\title{
Mechanisms of eukaryotic transcription
}

\author{
Miles A Pufall ${ }^{1 *}$ and Craig D Kaplan ${ }^{2^{*}}$
}

\section{Abstract \\ A report on the Cold Spring Harbor Laboratory \\ Mechanisms of Eukaryotic Transcription meeting, Cold Spring Harbor, New York, USA, August 27-31, 2013.}

Cold Spring Harbor Laboratory (CSHL) maintains its longstanding tradition of gathering researchers from all over the world to understand transcription and its regulation. Although powerful genomic techniques are now widely employed, with their implementation becoming more precise and sophisticated (for example, ChIP-seq and GRO-seq), structural and biochemical studies continue to advance our thinking. Sessions at the CSHL Mechanisms of Eukaryotic Transcription meeting were organized to focus on each step in transcription - initiation, elongation and termination - with others for signaling, regulation, chromatin, and genomics and systems biology. There were many highlights of the meeting, with threads of mechanistic insight and unexpected discoveries running throughout.

\section{Initiation mechanisms}

Eva Nogales (UC Berkeley, USA) began the meeting by describing recently published and highly intriguing electron microscopy (EM) structures of the human Pol II pre-initiation complex (PIC) and assembly intermediates, modeling positions for all major Pol II general transcription factors (GTFs). Kenji Murakami (Stanford University, USA) presented a cryo-EM structure of the yeast PIC with all GTFs bound. Predicted positions of GTFs were supported by cross-linking and mass spectrometry data computationally fit to a coarse-resolution model of the PIC. The human and yeast PIC structures showed a surprising number of differences, most centered around the location of Transcription factor II human (TFIIH) and positioning of downstream DNA.

\footnotetext{
* Correspondence: miles-pufall@uiowa.edu; cdkaplan@tamu.edu

'Department of Biochemistry, Carver College of Medicine, University of lowa, lowa City, IA 52242, USA

2Department of Biochemistry and Biophysics, Texas A\&M University, College Station, TX 77843, USA
}

Steve Hahn (Fred Hutchinson Cancer Research Center, USA) presented a characterization of the enzymatic activities of TFIIH. Purified TFIIH was shown to lack the $3^{\prime}-5^{\prime}$ helicase activity ascribed to its Ssl2 subunit (yeast $\mathrm{XPB}$ ) - the ATPase subunit required for promoter opening. In contrast, TFIIH possessed an Ssl2-dependent DNA translocase activity, supporting a model where TFIIH pumps DNA into the Pol II active site. Since TFIIH and upstream DNA are constrained in the PIC, its translocase activity torsionally strains DNA, resulting in unwinding within the Pol II active site.

Richard Ebright (Rutgers University, USA) addressed the basic enzymatic mechanism of initiation using Thermus thermophilus RNA polymerase (RNAP). In an amazing series of crystal structures, he revealed the first steps of transcript synthesis, including interactions of conserved RNAP residues with a pair of dNTP substrates prior to the first phosphodiester bond formation. Crystals containing several short RNA products also allowed direct observation of non-template DNA scrunching. Speaking of beautiful crystal structures, Patrick Cramer (Gene Center Munich, Germany) surprised the meeting participants with a new Pol I structure exhibiting a splayed DNA cleft that was too big for a double helix. This widening appeared to cause a change, compared with Pol II, which propagated to the active site that would prevent substrate binding. Conformational flexibility of this type has previously been observed for bacterial RNAPs but only now in a eukaryotic RNA polymerase. Cramer also presented a genomic analysis of how Nrd1/Nab3 non-coding RNA binding was likely embedded in sequence throughout the genome to terminate antisense transcription entering coding and non-coding regions, as well as divergent transcription from gene promoters.

\section{Elongation control}

Regulation of elongation focused on identification of new players. Heeyoun Bunch (Harvard Medical School, USA) used an immobilized template with a paused gene, human HSPA1B, to pull down TRIM28, a factor with previously identified roles in chromatin remodeling. 
Knockdown of the factor affected the expression of several paused genes, and redistributed Pol II to the gene body genome-wide, pointing to a pausing stabilization role. Building on previous work, Qiang Zhou (UC Berkeley, USA) found that AFF1, a central scaffolding protein for the super elongation complex (SEC), promotes HIV transcriptional elongation, at least in part, by enhancing $\mathrm{P}-\mathrm{TEFb}$ extraction from the 7SK snRNP by the viral encoded Tat protein.

Consistent with multiple functions of the AFF family (AFF1-4), Ali Shilatifard (Stowers Institute for Medical Research, USA) described work showing that P-TEFbcontaining complexes demonstrated higher levels of activity than P-TEFb alone towards the Pol II carboxyterminal domain, identifying SECs as the most active versions of P-TEFb. In addition to these factors, Monsef Benkirane (Institut Genetique Humaine, France) was able to show that the Integrator complex, which is better known for processing snRNA 3' ends, regulates NELFmediated Pol II pausing at protein coding genes. ChIP analyses show that Integrator associates with the transcriptional start site (TSS) of its target genes together with NELF, DSIF and Pol II. Knockdown of the Integrator catalytic subunit results in pause release and enhanced elongation by Pol II. However, Integrator also appears to be required for proper termination by Pol II. Thus, pause release and elongation are control points regulated by a number of factors.

Underscoring unexpected roles and mechanisms for known factors, work from David Price (University of Iowa, USA) and David Levens (National Cancer Institute, USA) examined the role of the oncogenic transcription factor Myc. David Levens demonstrated that Myc caused a global amplification of transcription in activated B cells with only a modest bonus at genes containing its cognate E-box binding site. David Price asserted that the genomic distribution of Myc suggested that the transcription machinery, rather than specific sequences, are the driving force for Myc occupancy. It remains to be seen how dispensable sequence-specific DNA binding is for Myc, and whether such promiscuity is widespread among other transcription factor families.

\section{Biochemistry of chromatin modifiers}

Two elegant biochemical studies revealed how reading multiple nucleosomes affects chromatin. Bing Li (UT Southwestern, USA) and Geeta Narlikar (UC San Francisco, USA) each used di-nucleosome substrates to probe how the modification status of one nucleosome affects the modification of another. Bing Li presented an intriguing model in which recognition of a dinucleosome and its spacing contributes to control of the $\mathrm{Rpd} 3$ deacetylase in yeast. Narlikar presented an exquisite mechanism of how multiple chromodomain containing proteins collaborate to form, maintain, or expand repressive heterochromatin using different factors (HP1 and Suv39/Clr4). She showed that despite recognizing the same methylated residue on nucleosomes (histone 3 lysine 9; H3K9), differential preferences for di- as opposed to tri-methyl states allowed the factors to collaborate rather than compete. It is likely that more interactions between differently modified nucleosomes and accessory proteins will reveal a level of regulation of transcription bewildering in both its subtlety and complexity.

\section{Unexpected partners in regulation}

Chromatin immunoprecipitation has become a standard tool in localizing transcription factors and chromatin modifications, with ChIP-seq allowing genome-wide analyses. Associations between co-localized factors have given great insight into functional relationships, while identifying new transcription factors. Colocalization of unexpected protein partners on target genes was evident in the discovery by Kathy Jones and colleagues (Salk Institute, USA) of $\alpha$-catenin functioning in a complex interplay with $\beta$-catenin as a transcriptional regulator when found on Wnt-pathway transcriptional targets. In addition, Jerry Workman (Stowers Institute for Medical Research, USA) described his group's identification of a Drosophila Hsc70 chaperone complex that localizes to specific genes and shows transcription factor-related effects. Both talks illustrated that the bounds of what factors might function in transcription need to be reconsidered.

In a striking piece of work, Rick Young (Whitehead Institute, USA) also used ChIP-seq signals of an active enhancer mark, H3K27Ac, in large (more than $10 \mathrm{~kb}$ ) domains to further identify their recently defined 'super enhancers' in a large panel of cell lines. Super enhancers contain high levels of numerous transcription factors and appear to be associated with master regulator genes required for cell-type identity. He also showed that these super enhancers in cancer lines are biomarkers for specific cancer types. Super enhancers appear to have bistable properties, and were significantly impaired by the inhibition of one cofactor, Brd4. How super enhancers are specified and evolve, and what relationships they have to cellular transformation remain to be elucidated.

\section{New techniques for deeper insight}

Despite the clear utility of ChIP-seq, several labs have pushed to enhance the technique. Frank Pugh (Penn State, USA) continued his work coupling traditional ChIP-seq with an exonuclease digestion step (ChIP-exo) to prune the $5^{\prime}$ ends of each DNA strand down to a site of crosslinking. This increases resolution, and enabled a detailed examination of GTF localization in human cells by Brian Venters from the Pugh lab (now at Vanderbilt University School of Medicine, USA) to identify core 
promoters. He found signatures of TATA elements and associated BRE sequences at most TFIIB ChIP-exo peaks, with elements exhibiting a stereotypical pattern among genes with highly diverse regulation. In an exciting development, David Auble (University of Virginia School of Medicine, USA) demonstrated that by measuring the ChIP signal of a transcription factor over a time course of exposure to crosslinking agent (seconds to minutes), the dynamics of site-specific binding could be inferred. This technique, termed crosslinking kinetic analysis, will help to explore the relationship between DNA-bound transcription factor turnover and transcriptional potency, competition and cooperativity among transcription factors, and gene expression timing. Furthermore, he demonstrated that a large dynamic range of signal is lost during long crosslinking times and how observed occupancy by ChIP for factors might be highly misleading.

An evolution of the Hi-C approach used in Bing Ren's lab (UC San Diego, USA), employing ultra-deep sequencing and new analysis methods to identify interactions between distant sequences, showed a surprising level of stability within what he termed 'topological domains' in human embryonic stem cells, which remained largely intact through development. Interestingly, two proteins thought to shape these boundaries, Cohesin and CTCF, were found to have distinct effects.

Two techniques illustrated subtleties of transcription only evident upon single-cell analyses. Single-cell RNA FISH (fluorescent in situ hybridization) experiments performed in Jane Mellor's lab (Oxford University, UK) pointed to a mechanistic link between antisense transcription and the behavior of sense-strand gene transcription. Her data suggested that sense and antisense transcription did not occur simultaneously, and that antisense transcription 'reset' the chromatin for transcription in the sense direction. The resulting back and forth resulted in increased noise or 'bursts' of sense transcription. Bursting was also observed in the early Drosophila embryo. In beautiful movies of live embryos shown by Michael Levine (UC Berkeley, USA), flickering or bursting transcription was observed for the promoter of the thisbe gene, which only weakly supports Pol II pausing, thus driving reporter expression. Promoters with strongly paused Pol II suppressed bursting, showing more even rates of mRNA production in similar experiments. These experiments indicate additional differences between strongly Pol II-pausing promoters and weakly pausing ones, which already have shown differences in stochasticity of on versus off states between embryonic nuclei. The core promoter thus may specify important additional properties of gene expression.

It is clear from these presentations, and many additional stories, that the CSHL Eukaryotic Transcription meeting occupies an important niche that draws from innovative genome scale experiments to study fundamental transcriptional mechanisms. We hope this focus continues and serves as a magnet to attract transcription researchers from all over the world.

\section{Abbreviations}

ChIP-seq: Chromatin immunoprecipitation followed by deep sequencing; GRO-seq: Global run on followed by deep sequencing; GTF: General transcription factor; H3K9: Histone 3 lysine 9; Pol I/Pol II: RNA polymerase I and II; P-TEFb: Positive transcription elongation factor b; RNAP: Bacterial RNA polymerase (RNAP); SEC: Super elongation complex; TFIIH: Transcription factor II human.

Competing interests

The authors declare that they have no competing interests.

Acknowledgements

Attendance of the meeting by the authors was supported by $\mathrm{NIH}$ grants R01GM097260 (CDK) and R00CA149088 (MAP).

Published: 30 September 2013

doi:10.1186/gb-2013-14-9-311

Cite this article as: Pufall and Kaplan: Mechanisms of eukaryotic transcription. Genome Biology 2013 14:311. 